\title{
On-ground flat-field calibration of the Metis coronagraph onboard the Solar Orbiter ESA mission
}

C. Casini, V. Da Deppo, P. Zuppella, P. Chioetto, A. Slemer, et al.

C. Casini, V. Da Deppo, P. Zuppella, P. Chioetto, A. Slemer, F. Frassetto, M. Romoli, F. Landini, M. Pancrazzi, V. Andretta, Y. De Leo, A. Bemporad, M. Fabi, S. Fineschi, F. Frassati, C. Grimani, G. Jerse, K. Heerlein, A. Liberatore, E. Magli, G. Naletto, G. Nicolini, M. G. Pelizzo, P. Romano, C. Sasso, D. Spadaro, M. Stangalini, T. Straus, R. Susino, L. Teriaca, M. Uslenghi, M. Casti, P. Heinzel, A. Volpicelli, "On-ground flat-field calibration of the Metis coronagraph onboard the Solar Orbiter ESA mission," Proc. SPIE 11852, International Conference on Space Optics - ICSO 2020, 118525B (11 June 2021); doi: 10.1117/12.2599945

Event: International Conference on Space Optics - ICSO 2021, 2021, Online Only 


\section{International Conference on Space Optics-ICSO 2020}

Virtual Conference

30 March-2 April 2021

Edited by Bruno Cugny, Zoran Sodnik, and Nikos Karafolas
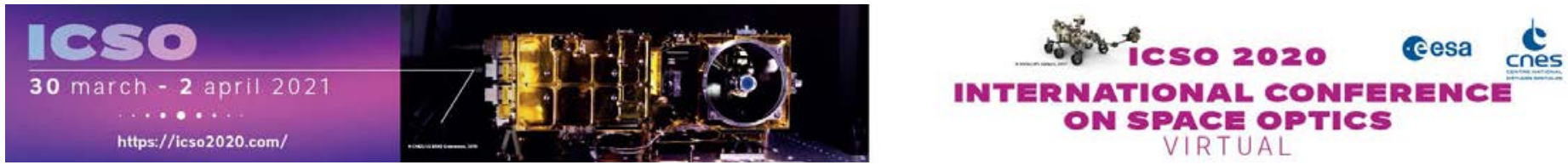

\section{On-ground flat-field calibration of the Metis coronagraph onboard the Solar Orbiter ESA mission}

\section{Cesa isoporecestings denes}




\title{
On-ground flat-field calibration of the Metis coronagraph on- board the Solar Orbiter ESA mission
}

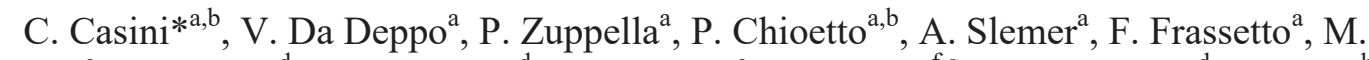

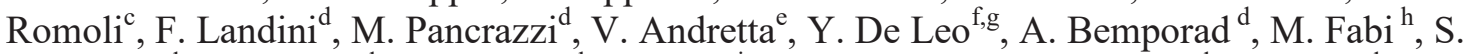
Fineschi $^{\mathrm{d}}$, F. Frassati ${ }^{\mathrm{d}}$, C. Grimani ${ }^{\mathrm{h}}$, G. Jerse ${ }^{\mathrm{i}}$, K. Heerlein ${ }^{\mathrm{g}}$, A. Liberatore ${ }^{\mathrm{d}}$, E. Magli ${ }^{\mathrm{d}}$, G.

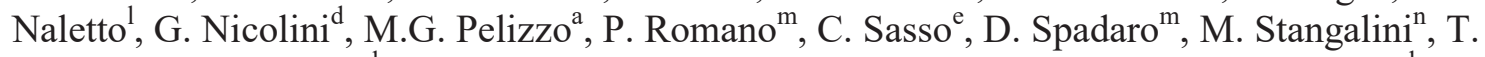

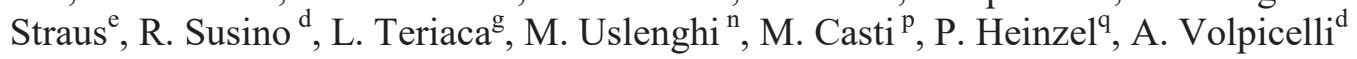

${ }^{\mathrm{a}} \mathrm{CNR}$ - IFN, Via Trasea 7, 35131, Padova, Italy; ${ }^{\mathrm{b}}$ CISAS, Centro di Ateneo di Studi e Attività Spaziali "Giuseppe Colombo", Via Venezia 15, 35131 Padova, Italy; ${ }^{\mathrm{c}}$ Dip.

di Fisica e Astronomia, Università di Firenze, Via Sansone, 1, 50019 Sesto Fiorentino (FI), Italy; ${ }^{\mathrm{d}} \mathrm{INAF}$ - Osservatorio Astrofisico di Torino, Via Osservatorio 20, Pino Torinese (To), Italy; ${ }^{\mathrm{e}}$ INAFOsservatorio Astronomico di Capodimonte, Salita Moiariello, 16 80131, Napoli, Italy; ${ }^{\mathrm{f}}$ Dip. di Fisica e Astronomia "Ettore Majorana" Università degli studi di Catania, Piazza università, 2, 95131 Catania, Italy; ${ }^{\mathrm{g}} \mathrm{MPS}$, Justus-von-Liebig-Weg 3, 37077 Göttingen, Germany; ${ }^{\mathrm{h}}$ Dip. Di Scienze Pure e Applicate, Università di Urbino, Via Santa Chiara, 27, Urbino, Italy; ${ }^{\mathrm{i}} \mathrm{INAF}-$ Osservatorio Astronomico di Trieste Via G.B. Tiepolo, 11 I, 34143 Trieste, Italy; ${ }^{1}$ Dip.

Di Fisica e Astronomia “Galileo Galilei”, Università di Padova, Via G. Marzolo, 8, 35131, Padova Italy; ${ }^{\mathrm{m}}$ INAF- Osservatorio Astrofisico di Catania, Via Santa Sofia, 78 I,95123 Catania, Italy; ${ }^{\mathrm{n}}$ Agenzia Spaziale Italiana, Via del Politecnico snc, 00133, Roma, Italy; ${ }^{\circ}$ INAF- Istituto di Astrofisica Spaziale e Fisica Cosmica, Via Alfonso Corti 1220133 , Milano, Italy; ${ }^{\mathrm{p}}$ The Catholic University of America at NASA's Goddard Space Flight Center, Greenbelt, MD 20771, USA;

${ }^{\mathrm{q}}$ Astronomical Institute of the Czech Academy of Sciences, Czech Republic

\begin{abstract}
Solar Orbiter, launched on February $9^{\text {th }} 2020$, is an ESA/NASA mission conceived to study the Sun. This work presents the embedded Metis coronagraph and its on-ground calibration in the 580-640 nm wavelength range using a flat field panel. It provides a uniform illumination to evaluate the response of each pixel of the detector; and to characterize the Field of View (FoV) of the coronagraph. Different images with different exposure times were acquired during the onground calibration campaign. They were analyzed to verify the linearity response of the instrument and the requirements for the FoV: the maximum area of the sky that Metis can acquire.
\end{abstract}

Keywords: Metis, Solar Orbiter, Coronagraph, Flat Field, Space Instrumentation

\section{INTRODUCTION}

The surroundings of the Sun have been studied since the invention of the coronagraph by Bernard Lyot in the 1930s [1]. A coronagraph is a telescope with an occulter (external or internal) that blocks the light from the photosphere, thus permitting the observation of the solar atmosphere. The first space coronagraph was created in the early $1960 \mathrm{~s}$ by Newkirk and Eddy who used a balloon to send their instrument to a height of $25 \mathrm{~km}$, where atmospheric scattering would be much smaller. Unfortunately, as the makers themselves stated, the instrument was not optimized for the reduction of scattered light from the edges of the optical elements, and therefore the observation failed [2].

*chiara.casini@pd.ifn.cnr.it; phone+393881649324 
It is strongly important to calibrate a space instrument on-ground and during the first phase of the mission. For the onground calibration, a flat field panel is used to simulate a uniform source. The recent orbital or sub-orbital space missions like SOHO, STEREO, HERSCHEL and Parker Solar Probe have something in common, they were calibrated in the same laboratory: the Solar Coronagraph Test Chamber (SCOTCH) facility at the Naval Research Laboratory (NRL, Washington, D.C., USA).

For the STEREO (SECCHI instrument) and SOHO (LASCO C3 instrument) missions, the flat field panel used for calibration consisted in two separated opal glass plates illuminated by a tungsten-halogen bulb with a power of 50 Watt. There was also a system that illuminated a field diaphragm in the lamp housing. Moreover, the opals have to be calibrated, for this purpose two instruments have been used: a spectroradiometer and a spot telephotometer. The correspondence between the two calibration methods is $10 \%[3,4]$.

Despite the previously cited space missions have already made observations of the Sun some open questions regarding many phenomena occurring in the atmosphere of out Star still remain e.g. how is the solar wind generated and accelerated in the interplanetary space? What is the origin and the development of the solar energetic particles [5]?

For this reason, the Solar Orbiter mission has been conceived. This spacecraft is different from the others because it goes close to the Sun as 0.28 AU while progressively inclining its orbital plane over the ecliptic. The coronagraph on-board Solar Orbiter is Metis, its characteristic is to have an inverse external occultation to reduce the thermal load while the coronagraph approaches the Sun.

This article describes the on-ground calibration of Metis using a flat field panel in visible light (580-640 nm). The resulting data were used to determine the performance of the instrument and its radiometric efficiency. The flat field panel has been used during the on-ground calibration for several purposes: evaluating the response of each pixel of the detector; and characterizing the response over the FoV of the coronagraph, i.e. determine the value of the vignetting along different radial profiles.

The next section describes in details the Metis coronagraph, while its calibration is presented in the third section.

\section{DESCRIPTION OF THE METIS CORONAGRAPH}

Metis is an aplanatic Gregorian telescope and in the following we describe the scheme and the optical path inside the instrument [6].

\subsection{Layout}

The light of the photosphere of the Sun enters Metis through the inverted external occulter (IEO) and it is rejected via $\mathrm{M}_{0}$ towards the entrance aperture, the $\mathrm{M}_{0}$ mirror acts as occulter as drawn in Figure 1.

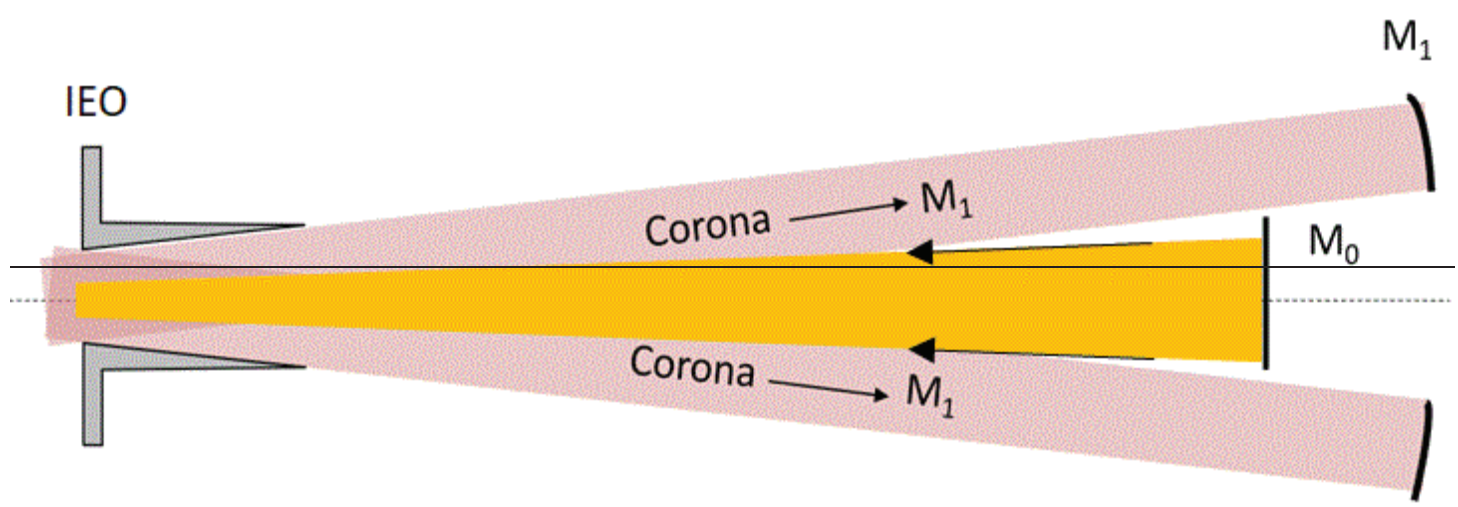

Figure 1 Schematic view of the Metis occultation. The light from the solar disk is back rejected by $\mathrm{M}_{0}$ (yellow). The light of the corona passes inside the coronagraph.

Between IEO and $\mathrm{M}_{0}$ there is a boom that consists of a black coated tube containing the Shield Entrance Aperture (SEA) diaphragm, which aims to reduce the diffused light inside the tube. The coronal signal is reflected by the mirror $\mathrm{M}_{1}$. The mirror $\mathrm{M}_{1}$ creates real images of the edges of IEO and $\mathrm{M}_{0}$; which are the main sources of the diffused light inside the 
coronagraph. This diffused light has to be blocked by the internal diaphragm system. The image of the edges of the IEO is blocked by the Internal Occulter (IO), while the image of the edges of the mirror $\mathrm{M}_{0}$ is eliminated by the Lyot Stop (LS). IO, LS the Field Stop (FS) are the elements between the mirror $\mathrm{M}_{1}$ and $\mathrm{M}_{2}$ as is drawn in Figure 2.

Then, after reflection on $\mathrm{M}_{1}$, the coronal light arrives at the mirror $\mathrm{M}_{2}$ and it is reflected in the direction of the dichroic beam-splitter, the Interferential Filter (IF). The IF is optimized for narrowband spectral transmission in the ultraviolet (UV, $121.6 \mathrm{~nm}$, HI Lyman- $\alpha$ ) and broadband spectral reflection in the visible (VIS, 580-640 nm).

The UV light path arrives on the UVDA (UV Detector Assembly). The visible light reflected by IF enters in a polarimetric unit to perform measurements of the polarization of the coronal radiation. The coronal light passes through a sequence of an optical collimating system (CD collimating doublet), a bandpass filter (BP), a linear polarization analyzer and is focused on the visible channel detector (Visible Channel Detector Assembly, VLDA). The polarization analyzer consists of a quarter-wave delay foil (QWP), a delay foil variable, liquid crystal (LCVR, liquid crystals variable retarder - Electro-optical Polarization Module Package, PMP), a linear polarizer (LP) as is drawn in Figure 2 .

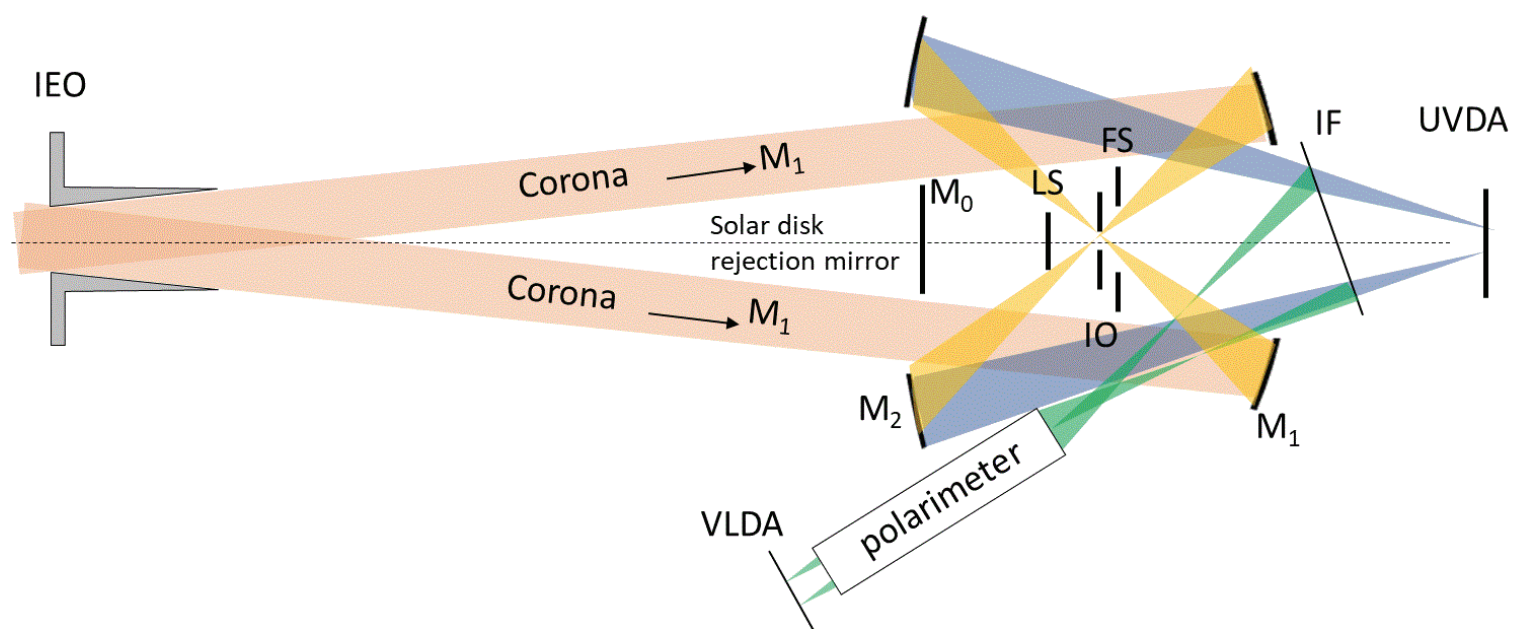

Figure 2 Schematic view of Metis coronagraph for both the UV and Visible Light channel.

\subsection{Ultraviolet (UV) and visible (VL) detector}

In this section the detectors of the Metis instrument are briefly described. The UV detector (UVDA) and Visible Light detector (VLDA) were developed by MPS ${ }^{1}$ specifically for the Solar Orbiter mission. The UVDA is located at the focal plane of the UV channel, it consists in a Micro Channel Plate (MCP), with a K-Br photocathode and is optically coupled, via an optical fiber cone, to an Active Pixel Sensor (APS, 1024 x 1024 pixels). The cone resizes the image of the focal plane on the detector so that it fits the sensor dimensions. Scaling is $2: 1$, so the $15 \mu \mathrm{m}$ pixel size of the APS detector results in an effective $30 \mu \mathrm{m}$ focal plane sampling element.

The VLDA is a camera sensitive to visible light. It is a complementary metal-oxide semiconductor (APS CMOS). Built very similar to the one used for the PHI instrument (developed by MPS), another instrument aboard Solar Orbiter. The VLDA is based on a CMOS APS sensor developed by CMOSIS ${ }^{2}$ with 2048 x 2048 pixels, which are $10 \mu \mathrm{m}$ in size.

\section{GROUND CALIBRATION METHODS AND RESULTS}

Ground calibrations are extremely important to characterize the response of an instrument. A flat field panel enabling a uniform illumination has been used to characterize the response of the instrument in all its field of view. This permits to calibrate the instrument optical elements together with the Metis detectors, as described in the section 2.2. The calibration images obtained with a flat field panel are hereafter called flat field images. This section is dedicated to the description of the experimental setup, and to the explanation of the analysis performed on the flat field images.

\footnotetext{
${ }^{1}$ Max-Planck-Institut fur Sonnensystemforschung, Gottingen, Germany

${ }^{2}$ http://www.cmosis.com/
} 


\subsection{Calibration test setup}

The flat field panel used for the on-ground calibration is model Spike-A flat fielder, it is composed by a LED matrix with several layers of diffusing material to obtain a quasi-uniform illumination (at $99 \%{ }^{3}$ ). Metis was positioned in front of the flat field panel as shown in Figure 3. The flat field panel must be positioned perpendicular to the optical axis of the telescope and must be powered at $9 \mathrm{~V}$. A lower voltage would lead to variations of brightness.

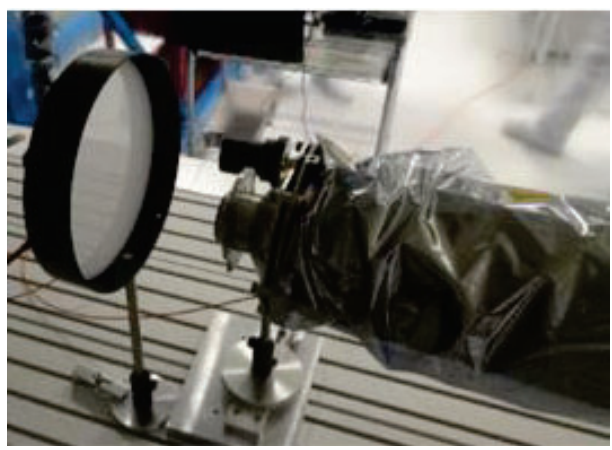

Figure 3 Optical layout of Metis in the laboratory calibration test set-up.

In Figure 4 the experimental scheme is represented. The flat field panel was positioned $10 \mathrm{~cm}$ from the Metis aperture, and a photodiode is set $8 \mathrm{~cm}$ from the centre of the IEO to check the flat field panel illumination performance. The photodiode measures the irradiance of the flat field panel, with an internal quantum efficiency of $100 \%$. In front of the photodiode there is a bandpass filter that is optimized for the visible wavelength: $580-640 \mathrm{~nm}$.

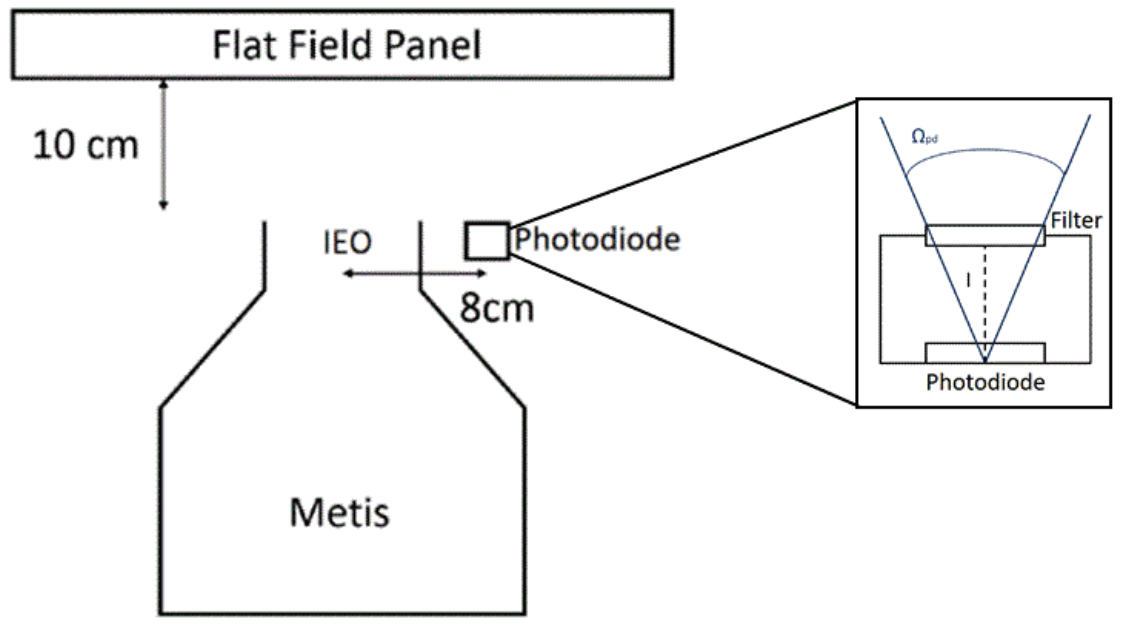

Figure 4 Diagram of the top and sectional view of the flat field panel, of the photodiode and of Metis with relative distances and with a zoom of the photodiode and its filter.

The image produced by this set up is shown in Figure 5. It measures the variations in the efficiency of the instrument due, in the case of Metis, mainly to the vignetting effect of the occultation system: $\mathrm{M}_{0}, \mathrm{IO}$, LS and FS.

To find the optimal exposure time, $\mathrm{t}_{\mathrm{opt}}$, and the intensity of the flat field panel (optimal voltage $\mathrm{V}_{\text {opt }}$ ) we studied the detected signal. If the signal is less than $2 / 3$ of the full well of the APS $(\sim 10000 \mathrm{DN})$, it is necessary to increase the intensity of the flat field panel. The flat field frames have been acquired at $20^{\circ} \mathrm{C}$. Preliminarily, images with an exposure between $0.25 \mathrm{~s}$ and $180 \mathrm{~s}$ were acquired, we found that the optimal configuration is the following: $\mathrm{t}_{\mathrm{opt}}=100 \mathrm{~s}$ and

${ }^{3}$ http://www.spike-a.com/flatfielders/ 
$\mathrm{V}_{\text {opt }}=11.5 \mathrm{~V}$. The next sub-sections are dedicated to the studies performed using these images, i.e. the study of the CCD response (3.2) and the validation of the Field of View requirements (3.3).

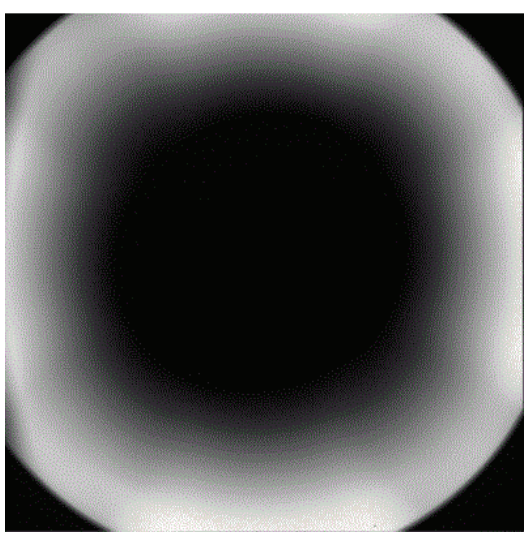

Figure 5. Image of the flat field panel after the subtraction of the dark.

\subsection{Preliminary analysis on the flat field images}

To know the response of the optical system, including the detector, an image of an extended and uniformly illuminated surface can be used. Firstly the four acquired flat field images were checked to see if there were any differences between nominally identical images.

The images were subtracted one by one obtaining the difference i.e. : FF2-FF1 (top left); FF3-FF2 (top right); FF4-FF3 (bottom left); and FF4-FF1 (bottom right) in Figure 6.
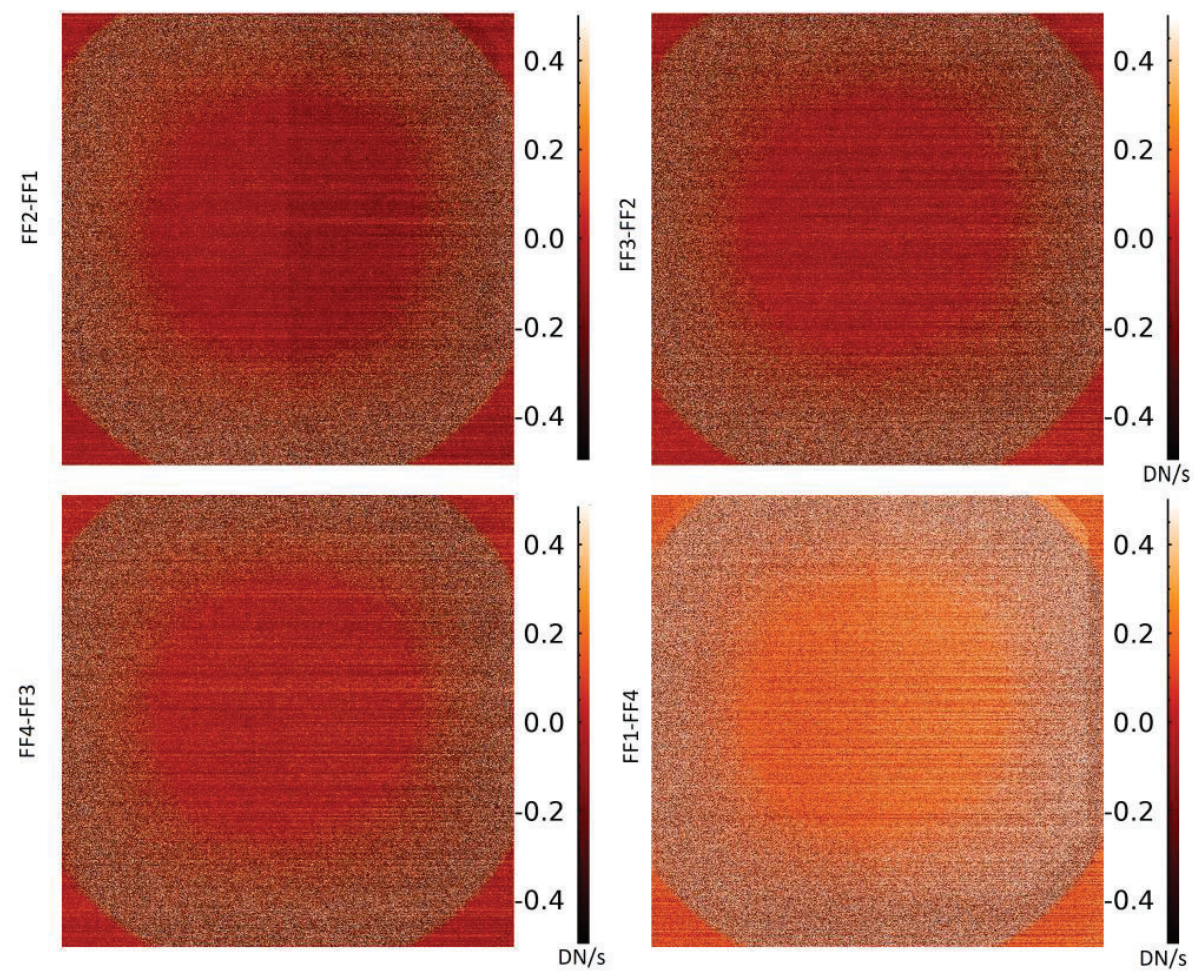

Figure 6. Flat field difference of 4 images acquired with $t_{\exp }=100$ s, in DN/s, i.e. divided by the integration time. 
The small differences between all the four frames have been studied. For the sake of clarity, we report hereafter only the study of the FF3-FF2 difference image, since similar features are observed on the other ones.

To this purpose two unidimensional plots (1D-plots) of the difference between two images divided by the integration time are represented in Figure 7, corresponding respectively to the central row (a), and to the central column (b). In the central part of the image, where the obscuration due to the occultation elements is placed, the noise level is lower. The noise level is due to the dark current and readout noise, while going to the edges the noise is increased with the increasing signal due to the presence of the light and the related shot noise.

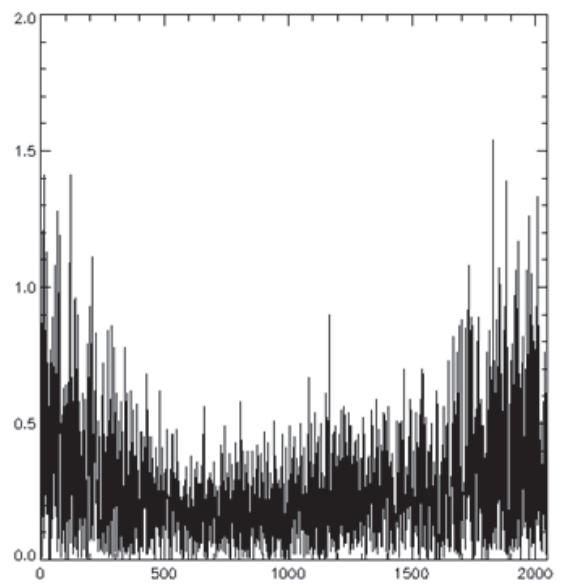

(a)

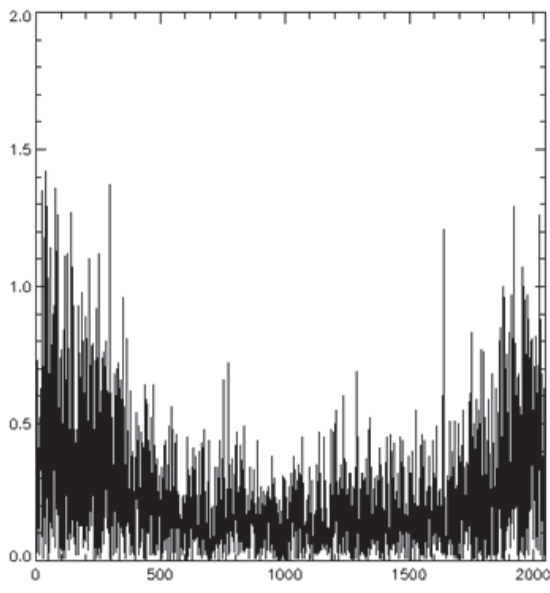

(b)

Figure 7. Unidimensional plots along for FF3-FF2 image in DN/s: in (a) row \#1024 ; in (b) column \#1024.

To demonstrate that the lower level of the noise is due to the absence of light, we also performed an increase of the contrast of the difference image FF3-FF2 (Figure 8a) and a contour plots of FF2 image (Figure 8b).

In both images we can notice that the light is blocked on the edges of the frame by the Field Stop and almost totally blocked in the centre thanks to the mirror $\mathrm{M}_{0}$, the Internal Occulter and the Lyot Stop. Besides, in the Figure 8a we can see the picture is less noisy at the edges and at the centre, this because we have just the dark current noise and the readout noise. Indeed, where more light arrives there is also the shot-noise to be considered. We can conclude that, considering all the noises, the images are nominally identical.

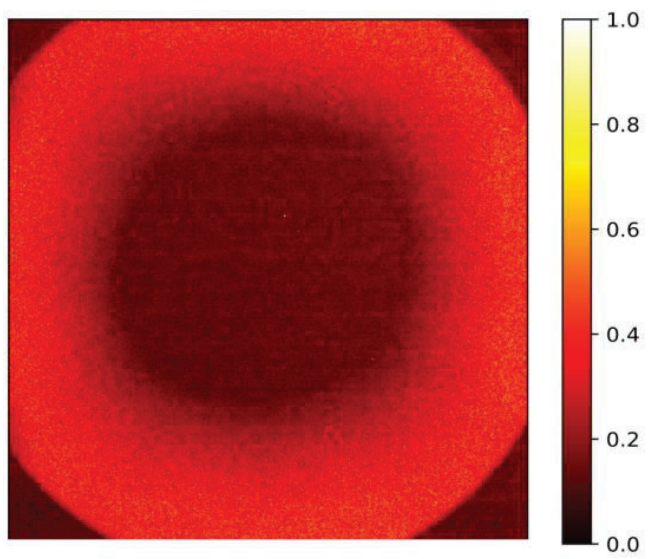

(a)

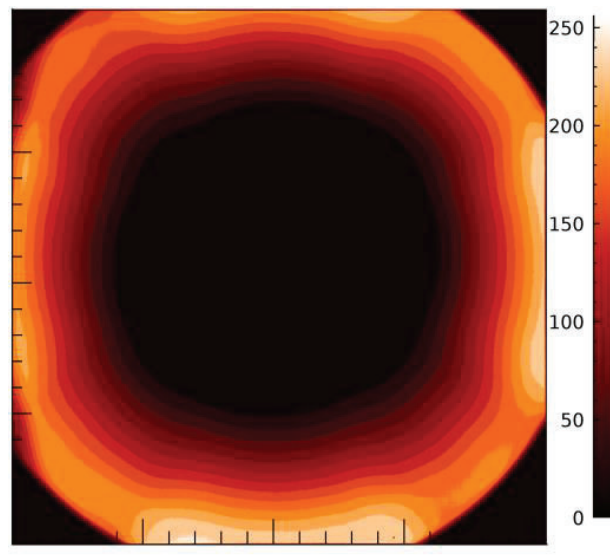

(b)

Figure 8. (a) increase of the contrast in the FF3-FF2 image; (b) contour plots of the FF2 image. 


\subsection{Verification of the Metis Field of View (FoV)}

The flat field images have been studied along some radial profiles as depicted in Figure 9. Lines at angles: $0^{\circ}-180^{\circ}$ (in blue); $45^{\circ}-225^{\circ}$ (in red); $90^{\circ}-270^{\circ}$ (in yellow); $135^{\circ}-315^{\circ}$ (in green). The theoretical vignetting function has been calculated through the raytracing software Zemax and compared with the measured one, see plots of Figure 10 where the measured profiles (different colours) are overlapped to Zemax calculated profiles in black.

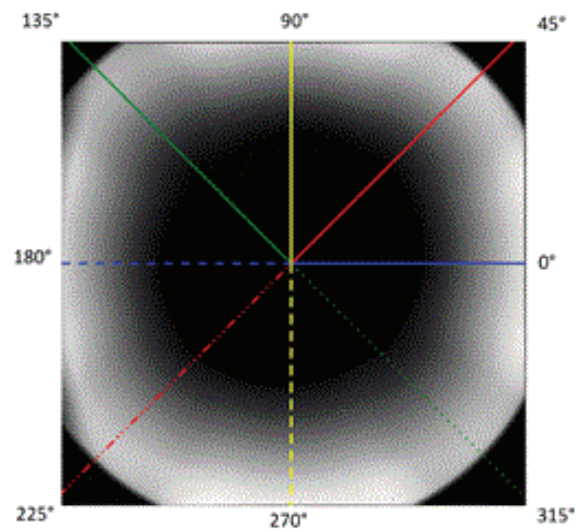

Figure 9 Flat filed image with lines from the centre to the edges at the angles $0^{\circ}-180^{\circ}$ (blue lines); $45^{\circ}-225^{\circ}$ (red lines); $90^{\circ}-270^{\circ}$ (yellow lines); $135^{\circ}-315^{\circ}$ (green lines).

All the curves were normalized to the maximum measured value in the radial direction $270^{\circ}$, and thus arbitrary units are used. The plots show that the field of view reaches $3.0^{\circ}$ for the directions $0^{\circ}-180^{\circ}$ and $90^{\circ}-270^{\circ}$, while it reaches $3.5^{\circ}$ for the directions $45^{\circ}-225^{\circ}$ and $135^{\circ}-315^{\circ}$. These plots also indicate the slope of the measured vignetting curve is consistent with the theoretical one.

It is worth to note that, with the available measurements, it is not possible to decouple the vignetting function from the efficiency of the coronagraph, we are bound to always study the product efficiency per vignetting.
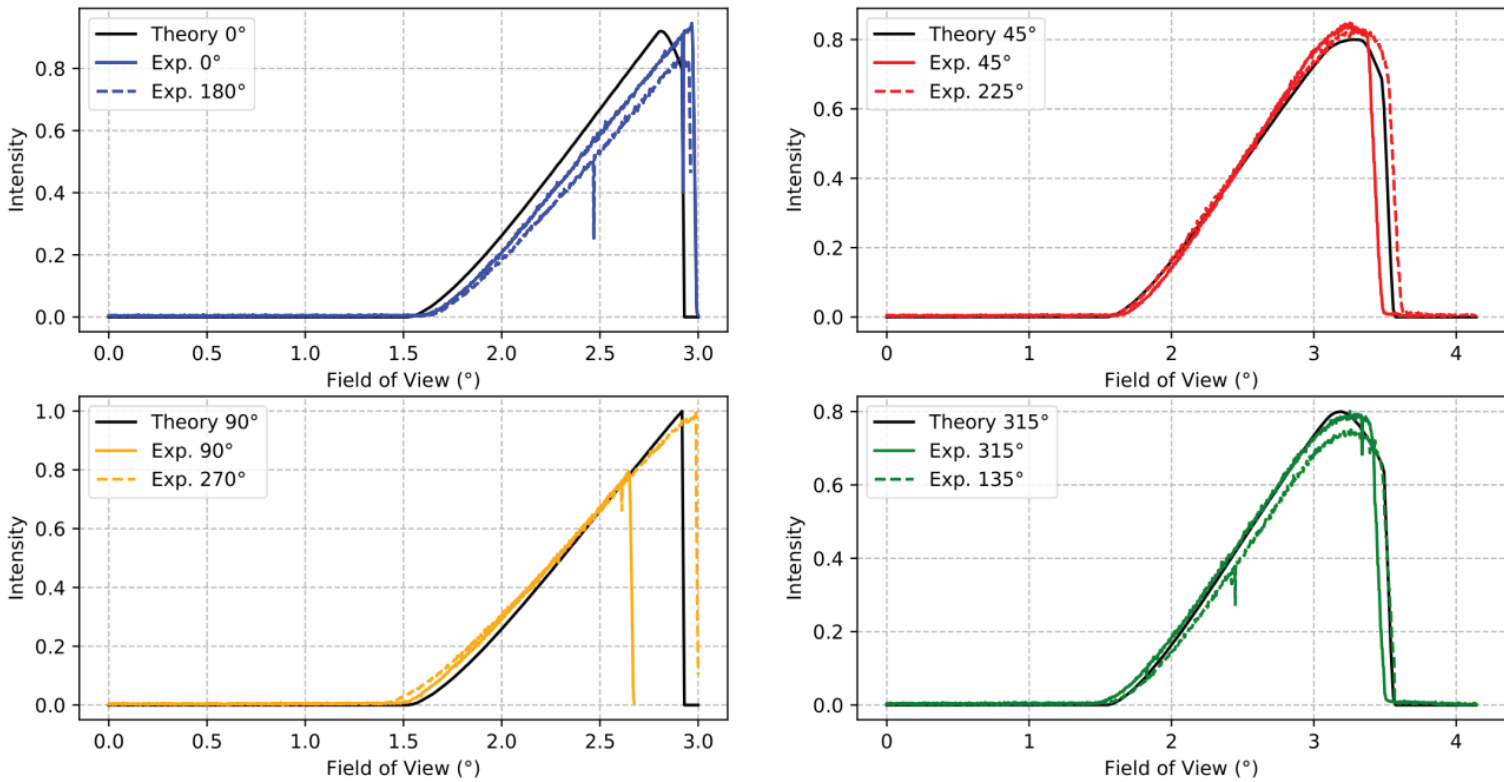

Figure 10. Trends of the flat field curve with varying angles: $0^{\circ}-180^{\circ}, 45^{\circ}-225^{\circ}, 90^{\circ}-270^{\circ}$ and $135^{\circ}-315^{\circ}$, normalized at the maximum value measured in the $270^{\circ}$ direction. The black lines, denoted with "Theory", are the ones calculated with the Zemax raytracing code, while the coloured "Exp" the measured ones. 


\section{CONCLUSIONS}

On-ground calibrations are fundamental for the correct interpretation of scientific images taken with space instrumentation, in particular for the coronagraph Metis on-board the Solar Orbiter mission, the calibration performed with the flat field panel is extremely important. A major result reported in this work is the verification of the instrument overall FoV. Some Metis optical components could have moved during the launch. Consequently, "investigations" are in progress in order to verify that these possible movements did not broadly impact the optical performances of the coronagraph.

Comparing the on-ground calibration and in-flight calibrations will give us, also, the opportunity to quantify the degradation of the system and to correct, from time to time and as possible, this effect.

\section{ACKNOWLEDGEMENT}

The author wish to thank Jeffrey Newmark for the useful support and for having share material on previous flat-field calibrations. Metis team thanks the former PI, Ester Antonucci, for leading the development of Metis until the final delivery to ESA.

The Metis program is supported by the Italian Space Agency (ASI) under the contracts to the co-financing National Institute of Astrophysics (INAF): Accordi ASI-INAF N. I-043-10-0 and Addendum N. I-013-12-0/1, Accordo ASI-INAF N. 2018-30-HH.0 and under the contracts to the industrial partners: ASI-TASI N. I-037-11-0 and ASI-ATI N. 2013-057I.0. The Metis team thanks Barbara Negri, Enrico Flamini, Marco Castronuovo of the Italian Space Agency and Roberto Della Ceca, Giuseppe Malaguti of the Istituto Nazionale di Astrofisica for their continuous support during the development of the coronagraph. A special thanks to Filippo Marliani of the European Space Agency for his dedication to the program and his high standard of excellence. The testing of Metis was performed by an industrial consortium constituted by OHB Italia S.p.A. (acting as Prime Contractor towards ASI), Thales Alenia Space Italia S.p.A. (Co-Prime Contractor with the specific responsibility of the instrument AIT). ALTEC has provided logistics and technical support for the INAF Optical Payload Systems. The primary and secondary mirrors were provided as Czech contribution to Metis; the mirror hardware development was possible thanks to the Czech PRODEX Programme. The UVDA assembly was provided as a German contribution to Metis, thanks to the financial support of DLR (grant 50 OT 1201). The VLDA assembly was provided under Contract 2013- 058-I.0 with the Italian Space Agency (ASI).

\section{REFERENCES}

[1] George Darwin Lecture, delivered by M. Bernard Lyot "A Study of the Solar Corona and Prominences Without Eclipses", Assoc. R.A.S. 580-585, (1939)

[2] Landini, F. \& Romoli, D. M. Analisi della luce diffusa nel coronografo UVCI di HERSCHEL. 133.

[3] Morrill, J. S. et al. Calibration of the Soho/Lasco C3 White Light Coronagraph. Sol Phys 233, 331-372 (2006).

[4] Howard, R. A. et al. Sun Earth Connection Coronal and Heliospheric Investigation. 56.

[5] Antonucci, E. et al. Metis: the Solar Orbiter visible light and ultraviolet coronal imager. A\&A 642, A10 (2020).

[6] Fineschi, S. et al. Optical design of the multi-wavelength imaging coronagraph Metis for the solar orbiter mission. Exp Astron, 49, 239-263 (2020). 